\title{
Near-Death Experiences Precipitated by Suicide Attempt: Lack of Influence of Psychopathology, Religion, and Expectations
}

\author{
Bruce Greyson, M.D. \\ University of Connecticut
}

\begin{abstract}
Near-death experiences (NDEs), transcendental or mystical events experienced on the threshold of death, have been speculated to arise from psychopathology or from pre-existing expectations of the dying process, although such speculations have never been tested. Sixty-one consecutive suicide attempters were interviewed in this empirical study of persons who would be expected to have a high rate of both psychopathology and coming close to death; $16(26 \%)$ reported near-death experiences (NDEs) as a result of the attempt. The group reporting NDEs and the group not reporting NDEs did not differ from each other in any parameters measuring psychopathology, religious background, or expectations of death and dying.
\end{abstract}

Reports of near-death experiences (NDEs), subjective events with transcendental or mystical elements that some persons experience when close to death, suggest that study of these phenomena may yield insights into the psychodynamics of our concepts of death and dying (Greyson, 1983). NDEs appear to induce substantial personality transformations (Noyes, 1980; Ring, 1984) and a significant reduction in suicidal ideation (Greyson, 1981). Despite the potential importance of near-death research for personality theory and suicide prevention,

Dr. Greyson is Associate Professor of Psychiatry at the University of Connecticut School of Medicine. At the time of this study, he was Assistant Professor of Psychiatry at the University of Michigan Medical School. Requests for reprints should be addressed to Dr. Greyson at the Department of Psychiatry, University of Connecticut Health Center, Farmington, CT 06030. 
however, few empirical studies of NDEs among suicide attempters have been conducted.

It has been speculated that NDEs arise as a manifestation of psychopathology (Greyson and Harris, 1987) or fulfillment of prior expectations of the dying process (Greyson, 1983). Although these hypotheses are contradicted by anecdotal data (Sabom, 1982), they have yet to be tested systematically. An analysis of the psychological meaning and value of the NDE must take into account the role of psychopathology and expectancy in these experiences.

I previously reported the incidence of NDEs among an unselected sample of consecutive suicide attempters to be $26 \%$, and the finding that NDEs among that sample were not related to age, gender, or race (Greyson, 1986). In the present paper, I describe these patients' prior evidence of psychopathology, religious background, and expectations of death and dying. I then describe the relation of those factors to the occurrence of NDEs following the suicide attempt, and discuss the implications of these data for hypotheses about the NDE.

\section{Method}

Sixty-one consecutive suicide attempters admitted to the University of Michigan Hospital were given a structured interview exploring demographic and historical data, including prior psychiatric treatment and suicidal behavior; family history of suicidal behavior; religious background; expectations of death and dying prior to the attempt; and features characteristic of NDEs occurring during the course of the suicide attempt or immediately thereafter. Details of patient selection are described elsewhere (Greyson, 1986). The Weighted Core Experience Index (WCEI), a graded unitary classification of NDE presence and "depth" (Ring, 1980), was imbedded within the interview.

The chi-squared statistic was used to test the hypotheses that NDEs would be associated with prior psychiatric treatment; with prior suicidal ideation or attempts; with family history of suicidal threats or attempts; with religion during childhood and at the time of the attempt; with prior close brushes with death; with prior knowledge of NDEs; with prior expectation that dying would be pleasant; and with prior expectation of continued existence after death. Two-tailed $t$ tests were used to test the hypotheses that NDEs would be associated with religiosity during childhood or at the time of the attempt. A p value < 0.05 was considered statistically significant. 


\section{Results}

As reported previously (Greyson, 1986), 16 of the 61 suicide attempters interviewed (26\%) reported experiences that scored 6 or higher on the WCEI, indicating the presence of an NDE.

Of the 61 attempters interviewed, $51(84 \%)$ reported prior treatment for a psychiatric or substance abuse problem. As shown in Table 1, prior psychiatric treatment did not differentiate those attempters who reported NDEs from those who did not.

Forty-three patients $(71 \%)$ reported prior suicide attempts; an additional 6 patients $(10 \%)$ reported prior suicidal ideation; and 12 patients (20\%) denied prior suicidal ideation. As shown in Table 1, prior suicidal ideation or behavior did not differentiate those attempters who reported NDEs from those who did not.

Twenty patients (33\%) reported a history of suicide attempts by another family member; an additional 3 patients $(5 \%)$ reported suicidal threats by another family member; and 38 patients $(62 \%)$ reported no suicidal threats or behavior among family members. As shown in Table 1, family history of suicide attempts or threats did not differentiate attempters who reported NDEs from those who did not.

Fifty-seven patients (93\%) described their family's religion during their childhood as Christian; 41 patients $(67 \%)$ described themselves as Christian at the time of the attempt. As shown in Table 1, neither childhood religion nor religion at the time of the attempt differentiated those attempters who reported NDEs from those who did not.

On a scale of "very religious" (=1), "moderately religious" $(=2)$, and "not at all religious" ( $=3$ ), the mean of the sample was 1.95 (S.D. = 0.78 ) for childhood religiosity and 2.31 (S.D. $=0.74$ ) for religiosity at time of the attempt. As shown in Table 1, neither religiosity during childhood nor religiosity at the time of the attempt differentiated attempters who reported NDEs from those who did not.

Thirty patients (49\%) reported having been previously near death, and 53 patients $(87 \%)$ reported some familiarity with the phenomenology of NDEs. As shown in Table 1, neither prior close brushes with death nor prior knowledge of NDEs differentiated attempters who reported NDEs from those who did not.

Twenty-three patients (38\%) reported that prior to the suicide attempt they had expected dying to be a predominantly pleasant experience; 7 patients $(12 \%)$ had expected it to be unpleasant; and 31 patients (51\%) had expected dying to be affectively neutral or had no expectation. As shown in Table 1, expectations of a pleasant or unpleasant 


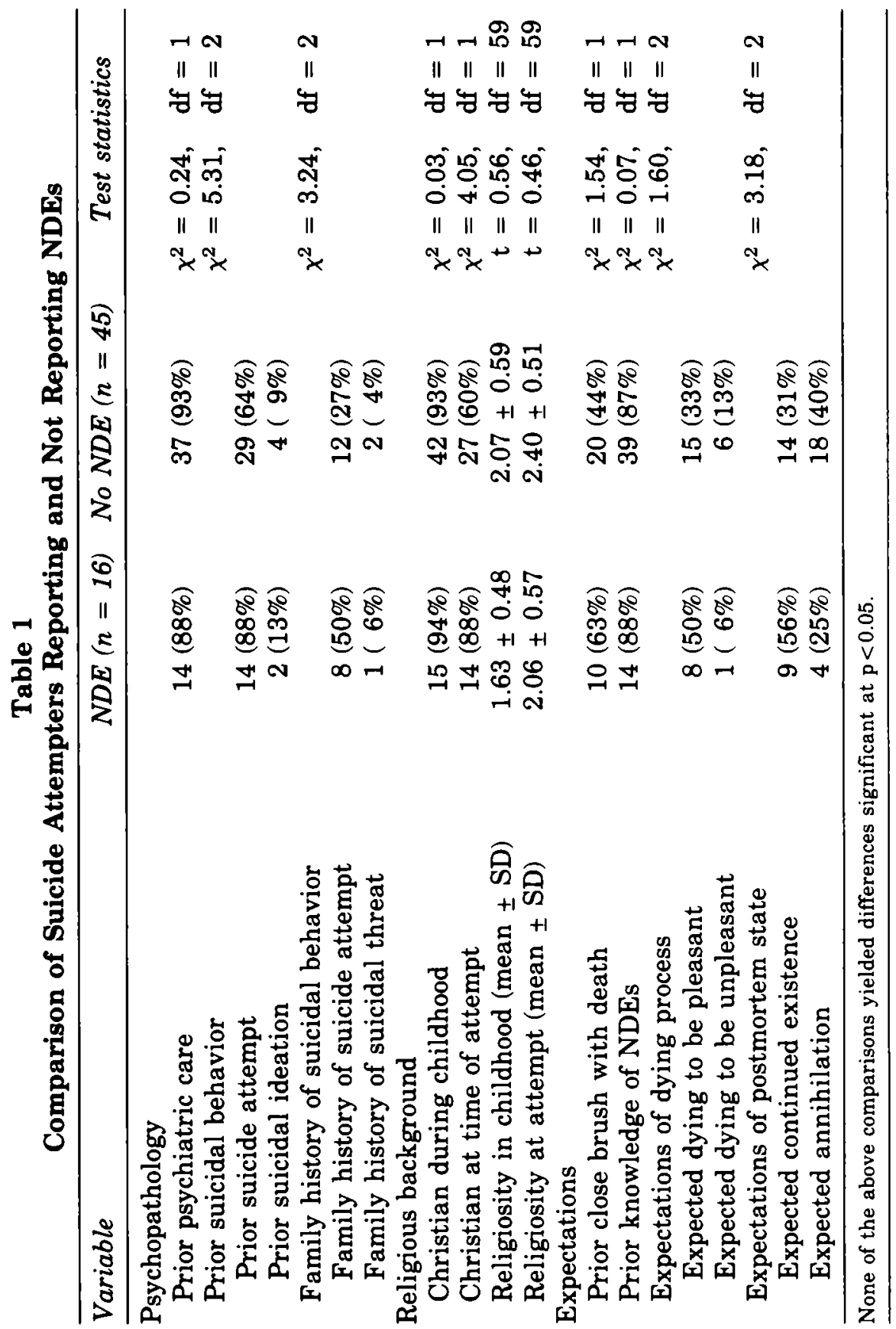


dying process did not differentiate attempters who reported NDEs from those who did not.

Twenty-three patients (38\%) reported that prior to the suicide attempt they had expected some sort of continued existence after bodily death; 22 patients (36\%) had expected total annihilation; and 16 patients $(26 \%)$ had no definite expectation. As shown in Table 1, expectations of postmortem continuation or annihilation did not differentiate attempters who reported NDEs from those who did not.

\section{Discussion}

The absence of any association in this study between NDEs and prior psychiatric treatment, prior suicidal behavior, or family history of suicidal behavior contradicts the hypothesis that NDEs reflect psychopathology. While that notion has been common among clinicians (Greyson and Harris, 1987), it has never before been tested.

The absence of any association between NDEs and religion or religiosity replicates the findings of other researchers (Ring, (1980; Sabom, 1982), contradicting the hypothesis that NDEs represent fantasies based on religious teachings.

The absence of any association between NDEs and prior close brushes with death or prior knowledge of NDEs contradicts the hypothesis that expectations are instrumental in eliciting an NDE. Bruce Greyson and Ian Stevenson (1980) had reported previously that no single feature of the NDE was associated with prior knowledge of NDEs. Likewise, the lack of association between NDEs and prior expectations of a pleasant dying process or of continued postmortem existence argues against a significant role for expectancy in the occurrence of NDEs.

These data suggest that the occurrence of NDEs is not influenced by psychopathology, by religious denomination or religiosity, or by experiencers' prior expectations of what death or dying might be like. While these findings do not lend positive support for any theory about the etiology of the NDE, they do cast some doubt on the hypothesis that NDEs arise primarily as a psychological defense.

\section{References}

Greyson, B. (1981). Near-death experiences and attempted suicide. Suicide and LifeThreatening Behavior, 11, 10-16. 
Greyson, B. (1983). The psychodynamics of near-death experiences. Journal of Nervous and Mental Disease, 171, 376-381.

Greyson, B. (1986). Incidence of near-death experiences following attempted suicide. Suicide and Life-Threatening Behavior, 16, 40-44.

Greyson, B., and Harris, B. (1987). Clinical approaches to the near-death experiencer. Journal of Near-Death Studies, 6, 41-52.

Greyson, B., and Stevenson, I. (1980). The phenomenology of near-death experiences. American Journal of Psychiatry, 137, 1193-1196.

Noyes, R., Jr. (1980). Attitude changes following near-death experiences. Psychiatry, 43, 234-242.

Ring, K. (1980). Life at death: $A$ scientific investigation of the near-death experience. New York, NY: Coward, McCann and Geoghegan.

Ring, K. (1984). Heading toward omega In search of the meaning of the near-death experience. New York, NY: Morrow.

Sabom, M.B. (1982). Recollections of death: A medical investigation. New York, NY: Harper and Row. 